Article

\title{
An Ultra-High-Q Lithium Niobate Microresonator Integrated with a Silicon Nitride Waveguide in the Vertical Configuration for Evanescent Light Coupling
}

\author{
Jianhao Zhang ${ }^{1,2}$, Rongbo Wu ${ }^{1,2}$, Min Wang $3,4, * \mathbb{C}$, Youting Liang ${ }^{3,4}$, Junxia Zhou ${ }^{3,4}$, Miao Wu ${ }^{3,4}$, \\ Zhiwei Fang ${ }^{3,4}$, Wei Chu ${ }^{4, *}$ (i) and Ya Cheng $1,3,4,5,6,7,8, *$ (i)
}

1 State Key Laboratory of High Field Laser Physics and CAS Center for Excellence in Ultra-Intense Laser Science, Shanghai Institute of Optics and Fine Mechanics, Chinese Academy of Sciences, Shanghai 201800, China; jhzhang@siom.ac.cn (J.Z.); rbwu@siom.ac.cn (R.W.)

2 Center of Materials Science and Optoelectronics Engineering, University of Chinese Academy of Sciences, Beijing 100049, China

3 State Key Laboratory of Precision Spectroscopy, East China Normal University, Shanghai 200241, China; 15253172638@163.com (Y.L.); 52180920026@stu.ecnu.edu.cn (J.Z.); wumiao1993@126.com (M.W.); zwfang@phy.ecnu.edu.cn (Z.F.)

4 The Extreme Optoelectromechanics Laboratory (XXL), School of Physics and Electronic Science, East China Normal University, Shanghai 200241, China

5 Collaborative Innovation Center of Extreme Optics, Shanxi University, Taiyuan 030006, China

6 Collaborative Innovation Center of Light Manipulations and Applications, Shandong Normal University, Jinan 250358, China

updates

Citation: Zhang, J.; Wu, R.; Wang, M.; Liang, Y.; Zhou, J.; Wu, M.; Fang, Z.; Chu, W.; Cheng, Y. An Ultra-High-Q Lithium Niobate Microresonator Integrated with a Silicon Nitride Waveguide in the Vertical Configuration for Evanescent Light Coupling. Micromachines 2021, 12, 235. https://doi.org/10.3390/ mi12030235

Academic Editor: Andrea Ehrmann

Received: 1 February 2021

Accepted: 21 February 2021

Published: 25 February 2021

Publisher's Note: MDPI stays neutral with regard to jurisdictional claims in published maps and institutional affiliations.

Copyright: (C) 2021 by the authors. Licensee MDPI, Basel, Switzerland. This article is an open access article distributed under the terms and conditions of the Creative Commons Attribution (CC BY) license (https:/ / creativecommons.org/licenses/by/ $4.0 /)$.
7 CAS Center for Excellence in Ultra-Intense Laser Science, Shanghai 201800, China

8 Shanghai Research Center for Quantum Sciences, Shanghai 201315, China

* Correspondence: mwang@phy.ecnu.edu.cn (M.W.); wchu@phy.ecnu.edu.cn (W.C.); ya.cheng@siom.ac.cn (Y.C.)

\begin{abstract}
We demonstrate the hybrid integration of a lithium niobate microring resonator with a silicon nitride waveguide in the vertical configuration to achieve efficient light coupling. The microring resonator is fabricated on a lithium niobate on insulator (LNOI) substrate using photolithography assisted chemo-mechanical etching (PLACE). A fused silica cladding layer is deposited on the LNOI ring resonator. The silicon nitride waveguide is further produced on the fused silica cladding layer by first fabricating a trench in the fused silica while using focused ion beam (FIB) etching for facilitating the evanescent coupling, followed by the formation of the silicon nitride waveguide on the bottom of the trench. The FIB etching ensures the required high positioning accuracy between the waveguide and ring resonator. We achieve Q-factors as high as $1.4 \times 10^{7}$ with the vertically integrated device.
\end{abstract}

Keywords: lithium niobate microring resonator; silicon nitride waveguide; photolithography assisted chemo-mechanical etching

\section{Introduction}

Lithium niobate (LN) has long been recognized as an important material platform for integrated photonic devices because of its wide transparent window, high nonlinear coefficient, and excellent electro-optical property [1]. In particular, the latest advancement of the fabrication technologies of high-quality photonic micro- and nanostructures on lithium niobate on insulator (LNOI) has further promoted the development of integrated photonics on the LNOI platform. The building block photonic structures, such as microresonator and optical waveguide, are typically fabricated on LNOI substrate using either maskless focused ion beam (FIB) milling or lithographic processing involving argon ion milling [2-11]. Meanwhile, a chemo-mechanical etching process (termed photolithography assisted chemo-mechanical etching (PLACE) hereafter) has been developed to achieve ultra-high surface smoothness, resulting in high-quality (high-Q) microdisk resonator 
with a Q-factor of $4 \times 10^{7}$ and ultra-low-loss optical waveguide with a propagation loss of $0.03 \mathrm{~dB} / \mathrm{cm}$ [12-14]. So far, a broad range of nonlinear optical processes have been demonstrated with the ultra-high-Q LN microresonators that were fabricated using the PLACE technology, ranging from optomechanics [15] and optical frequency comb [16] to nonlinear frequency conversion [17] and on-chip micro-disk lasing.

Light must be efficiently coupled into the microresonaotors using either a fiber taper [18] or an integrated optical waveguide [19] in order to excite the nonlinear optical effects. The on-chip integration of the microresonator and the coupling optical waveguide provides an efficient means for up scaling of the photonic integration circuits (PICs), which is critical for some applications, such as photonic computation and quantum information processing [20], etc. However, in the PLACE scheme, the low-loss optical waveguides and high $\mathrm{Q}$ microresonators are both generated using the chemical-mechanical polishing (CMP) technique. In the CMP process, it is required that the distance between the closely located photonic structures should be on the micrometer scale, but not the nanometer scale; otherwise, the LNOI in the narrow gap between the neighboring structures cannot be efficiently removed by polishing. In this case, the lateral evanescent coupling between a microresonator and a waveguide is difficult to achieve, simply because of the fact that the evanescent coupling in the visible and near infrared ranges requires the gap width to be in the order of a few hundred nanometers. For this reason, on-chip evanescent coupling has not been realized between an optical waveguide and a microresonator fabricated using the PLACE technique.

Here, we overcome the difficulty by utilizing a vertical coupling scheme between a crystalline $\mathrm{LN}$ microring resonators and a silicon nitride $\left(\mathrm{Si}_{3} \mathrm{~N}_{4}\right)$ waveguide. $\mathrm{Si}_{3} \mathrm{~N}_{4}$ is also considered to be an attractive candidate for monolithic integration of photonic circuits because of its low propagation loss. Importantly, $\mathrm{Si}_{3} \mathrm{~N}_{4}$ has a refractive index that is close to that of $\mathrm{LN}$, which makes it easy to fulfill the phase matching condition between the $\mathrm{Si}_{3} \mathrm{~N}_{4}$ waveguide and LN microresonator. We characterized the integrated device by measuring the Q-factor of the fabricated LN microresonator, and demonstrated the coupling control by varying the thickness of the $\mathrm{SiO}_{2}$ cladding layer.

\section{Materials and Methods}

The microring resonator is fabricated on a commercially available $\mathrm{x}$-cut LNOI wafer with a thickness of $900 \mathrm{~nm}$ (NANOLN, Jinan Jingzheng Electronics Co., Ltd., Jinan, Shandong, China). The $\mathrm{LN}$ thin film is bonded to a $2 \mu \mathrm{m}$-thick $\mathrm{SiO}_{2}$ layer supported by a $500-\mu \mathrm{m}$-thick LN substrate. Figure 1a depicts the configuration of the LNOI wafer, followed by the schematic of process flow, as shown in Figure 1b-k. In general, the fabrication procedures include: (1) the deposition of a thin layer of chromium $(\mathrm{Cr})$ with a thickness of $400 \mathrm{~nm}$ on the surface of the LNOI by magnetron sputtering (Figure 1b); (2) spaceselective ablation of a Cr layer coated on top of the LNOI to generate the pattern of the microring resonator using a focused femtosecond laser beam (Figure 1c). In this step, the femtosecond laser ablation was conducted by a commercial laser system (Pharos, LightConversion, Lithuania) at a repetition rate of $500 \mathrm{kHz}$ and a scan speed of $40 \mathrm{~mm} / \mathrm{s}$. The center wavelength of the femtosecond laser was $1030 \mathrm{~nm}$, and the pulse width was set to be $\sim 270$ fs. A $100 \times$ objective lens (M Plan Apo NIR, Mitutoyo, Japan) with a numerical aperture (NA) of 0.7 was employed to pattern the Cr layer in order to obtain a high ablation resolution. Femtosecond laser ablation was carried out by translating the sample with a three-dimensional (3D) motion stage (ABL1500-ANT130, Aerotech Inc., USA); (3) etching of the LNOI layer by CMP (Figure 1d). In this step, the LN without being covered by the $\mathrm{Cr}$ mask will be completely removed, while the LN protected by Cr mask will survive from the CMP because of the high hardness of $\mathrm{Cr}$; (4) removal of the residual Cr mask left on the surface of LNOI by chemical wet etching, and further eliminate the roughness by a second CMP process (Figure 1e); (5) the deposition of the $\mathrm{SiO}_{2}$ film on the LNOI waveguide to form the cladding layer by plasma enhanced chemical vapor deposition (PECVD) (Figure 1f); (6) polishing the surface of $\mathrm{SiO}_{2}$ cladding layer with the third CMP 
(Figure 1g); (7) patterning of the $\mathrm{SiO}_{2}$ layer while using focused ion beam (FIB) etching (Figure 1h). In particular, the depth of the etched trench can be controlled with an accuracy of $\sim 1 \mathrm{~nm}$ using the FIB etching; (8) the deposition of a $\mathrm{Si}_{3} \mathrm{~N}_{4}$ film on the $\mathrm{SiO}_{2}$ layer by PECVD to fill the trench fabricated in the $\mathrm{SiO}_{2}$ cladding layer (Figure 1i); (9) removing the $\mathrm{Si}_{3} \mathrm{~N}_{4}$ above the $\mathrm{SiO}_{2}$ layer with the fourth CMP (Figure 1j); and, (10) patterning of the $\mathrm{Si}_{3} \mathrm{~N}_{4}$ film in the trench using FIB etching to form the waveguide (Figure 1k). More details of the femtosecond laser micromachining of $\mathrm{Cr}$, the CMP processing, and the FIB etching can be found elsewhere [12-14]. Figure 11 shows a schematic 3D view of the hybrid LN and $\mathrm{Si}_{3} \mathrm{~N}_{4}$ coupling structure.

(a)

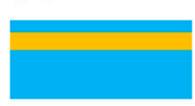

(e)

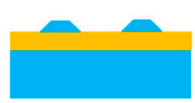

(i)

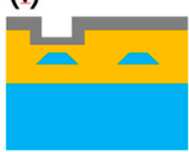

(b)

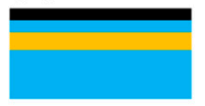

(f)

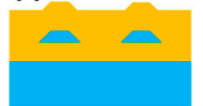

(j)

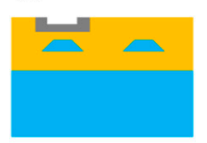

(c)

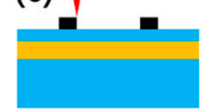

(g)

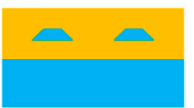

(k)

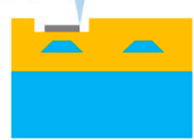

(d)

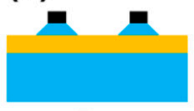

(h)
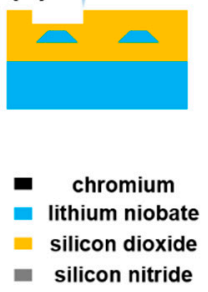

(I)

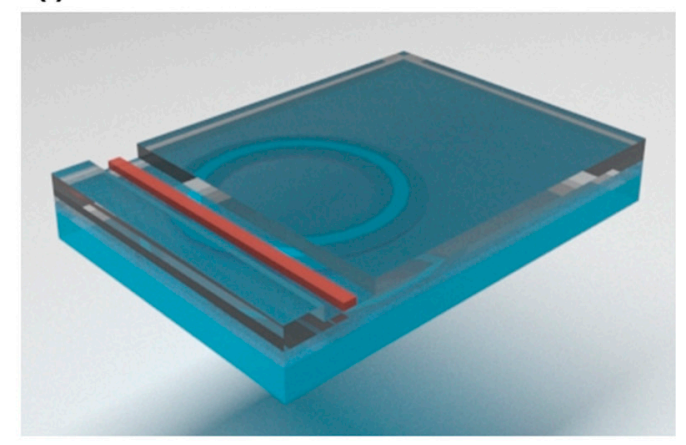

Figure 1. (a-k) Process flow of fabricating a lithium niobate (LN) microring resonator coupling with a silicon nitride $\left(\mathrm{Si}_{3} \mathrm{~N}_{4}\right)$ waveguide in the vertical configuration. (1) Three-dimensional (3D) diagram of the coupling structure.

\section{Results}

Figure 2a shows the top-view scanning electron micrograph (SEM) of the vertically coupled $\mathrm{LN}$ microring and $\mathrm{Si}_{3} \mathrm{~N}_{4}$ waveguide. The profiles of the microring buried beneath $\mathrm{SiO}_{2}$ layer can be distinguished in the SEM image. The radius of the LN microring is $50 \mu \mathrm{m}$. The cross section of the LN microring, as indicated by the blue dashed area in Figure 2a, is shown in Figure $2 b$. In the current design, the LN microring has a trapezoidal cross-section with a top width of $2.5 \mu \mathrm{m}$, a bottom width of $7.5 \mu \mathrm{m}$, and a height of $800 \mathrm{~nm}$, which is covered by a $1.5-\mu \mathrm{m}$-thick $\mathrm{SiO}_{2}$ cladding layer. The cross section of the coupling area (yellow dashed area in Figure 2a) is shown in Figure 2c. The three-layer structure, i.e., LN, $\mathrm{SiO}_{2}$ and $\mathrm{Si}_{3} \mathrm{~N}_{4}$ from bottom to top, can be clearly seen. The thickness of the PECVD $\mathrm{SiO}_{2}$ layer was reduced from $1.5 \mu \mathrm{m}$ to $600 \mathrm{~nm}$ by FIB etching for evanescent light coupling. The rib $\mathrm{Si}_{3} \mathrm{~N}_{4}$ waveguide was fabricated by FIB etching with a width of $3.5 \mu \mathrm{m}$ and a rib height of $550 \mathrm{~nm}$.
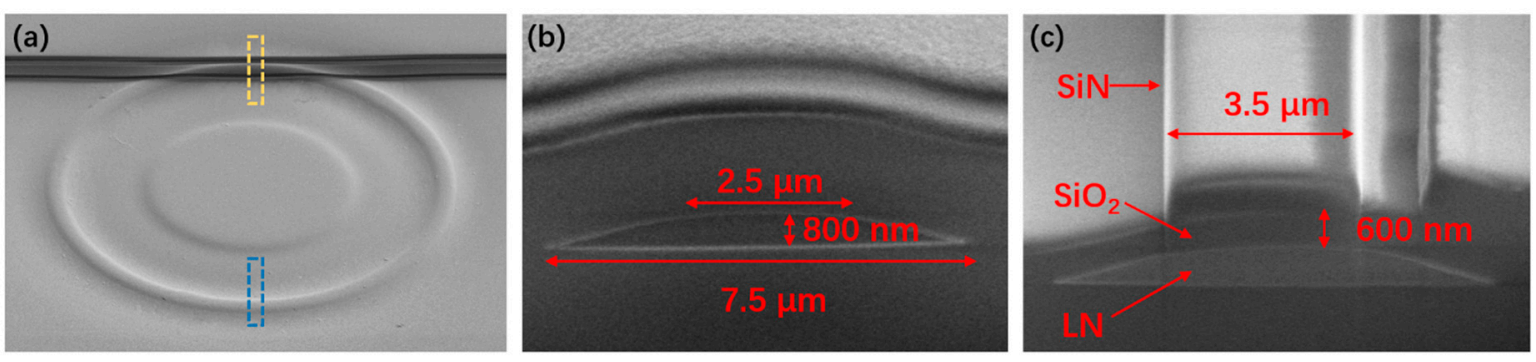

Figure 2. (a) Top view scanning electron micrograph (SEM) image of $\mathrm{LN}$ microring resonator and $\mathrm{Si}_{3} \mathrm{~N}_{4}$ waveguide. (b) Sectional view SEM image of the structure at the location of the bule dashed box in (a). (c) Sectional view SEM image of the structure at the location of the yellow dashed box in (a).

Furthermore, we measured the surface roughness for both the $\mathrm{LN}$ microring resonator fabricated by $\mathrm{CMP}$ and the trench in $\mathrm{SiO}_{2}$ layer (i.e., the bottom of the trench) generated 
by FIB etching that are critical in obtaining high $\mathrm{Q}$ factors. Figure 3a,b present the SEM images of fabricated LN microring after CMP (step e in Figure 1) and the etched trench of the $\mathrm{SiO}_{2}$ layer using FIB etching (step $\mathrm{h}$ in Figure 1). Using an atomic force microscope (AFM), we measured the surface root-mean-square roughness $(\mathrm{Rq})$ in the areas that are indicated by the red squares in Figure $3 a, b$ respectively. An ultralow surface roughness of $\mathrm{Rq} \sim 0.45 \mathrm{~nm}$ can be achieved by the CMP processing, while a slightly higher roughness of $\mathrm{Rq} \sim 1.22 \mathrm{~nm}$ was achieved after FIB etching. Based on the smooth surface morphology, we can expect a high-Q LN microring resonator.
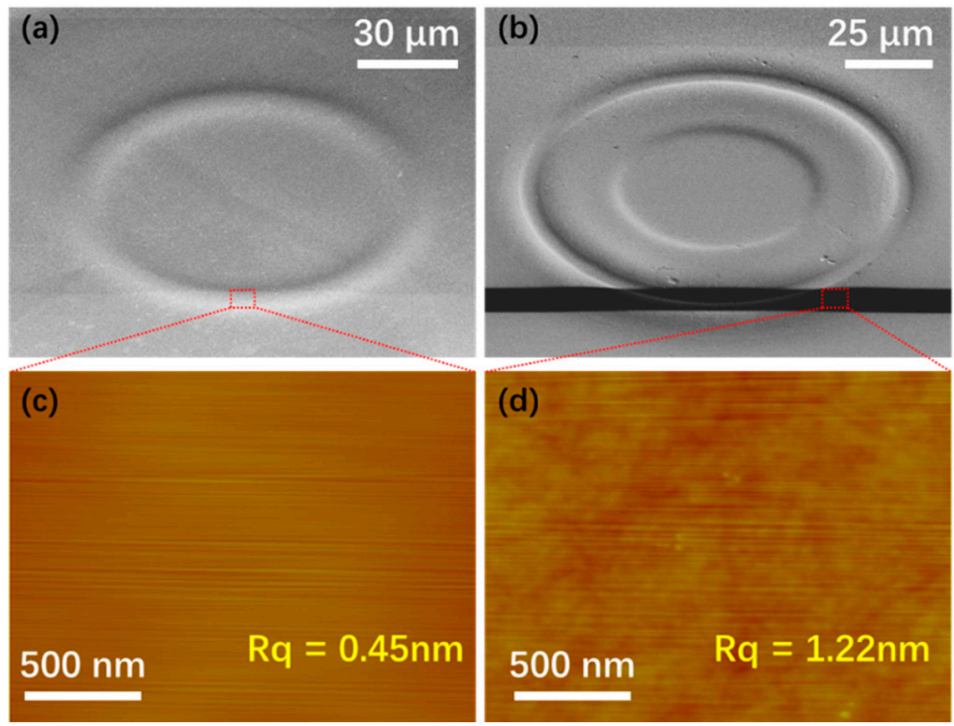

Figure 3. (a) Top view SEM image of the fabricated LN microring resonator. (b) Top view SEM image of the trench fabricated in the $\mathrm{SiO}_{2}$ cladding layer. (c,d) Atomic force microscope (AFM) images of the surfaces of the microring in (a) and the trench in (b) respectively.

We used an experimental setup to examine the coupling effect of the configuration and characterize the optical mode structure of the LN ring resonator, as schematically shown in Figure 4. A tunable laser (TLB 6728, New Focus Inc., San Jose, CA, USA) was employed to couple light into and out of $\mathrm{Si}_{3} \mathrm{~N}_{4}$ waveguide through the lensed fiber with a taper angle of $90^{\circ}$. In order to enhance the detection signal, the tunable laser was boosted by an erbiumytterbium-doped fiber amplifier (EYDFA, Golight, Inc., Culbertson, NE, USA) before coupling into the $\mathrm{Si}_{3} \mathrm{~N}_{4}$ waveguide. The linewidth of the tunable laser is $200 \mathrm{kHz}$. The polarization of the pump laser was adjusted by an in-line fiber polarization controller. A photodetector measured the transmission of resonant mode (New focus 1811-FC-AC, Newport Inc., Irvine, CA, USA). We used an arbitrary waveform generator (AFG3052C, Tektronix Inc., Beaverton, DC, USA) to synchronize the tunable laser and oscilloscope signals.

Figure 5a shows the transmission spectrum for the wavelength range from $1537 \mathrm{~nm}$ to $1562 \mathrm{~nm}$. The free spectral range (FSR) of the microresonator was measured to be 3.34 $\mathrm{nm}$. A pair of the splitting whispering-gallery modes at the resonant wavelength around $1543.52 \mathrm{~nm}$ was chosen for the measurement of the Q-factor by fitting with a Lorentz function. The $\mathrm{Q}$ factors were measured to be $1.49 \times 10^{7}$ and $1.09 \times 10^{7}$, respectively, as indicated by the Lorentz curves shown in Figure $5 \mathrm{~b}$. The $\mathrm{Q}$ factors vary from $2.9 \times 10^{6}$ to $1.49 \times 10^{7}$, which is mainly caused by the different coupling condition of the whispering gallery modes. The high Q-factor of the LN microresonator indicates that the fabricated device with the vertical integration configuration functions effectively for evanescent light coupling between the $\mathrm{LN}$ microring and the $\mathrm{Si}_{3} \mathrm{~N}_{4}$ waveguide. 


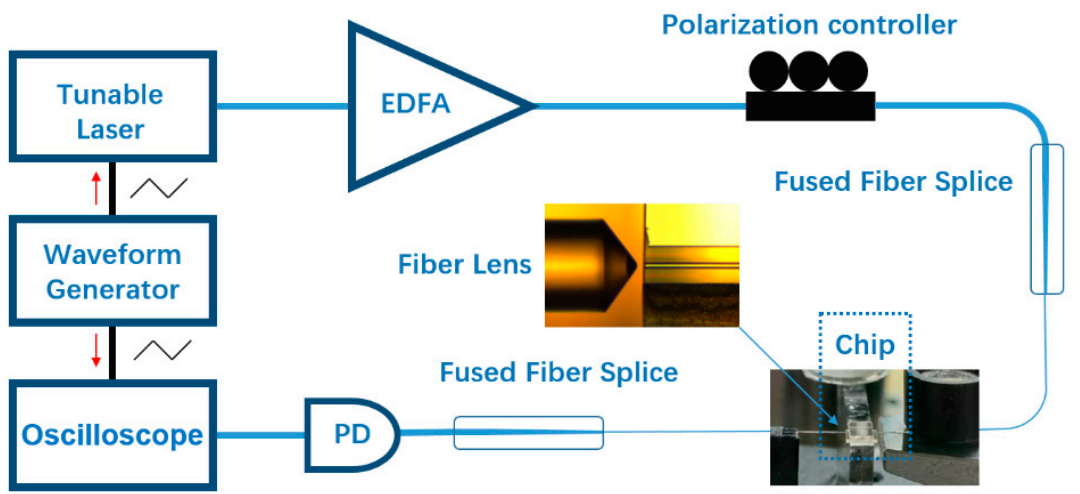

Figure 4. Schematic of experimental testing setup. Inset: optical micrograph of the fiber lens coupling with the $\mathrm{Si}_{3} \mathrm{~N}_{4}$ waveguide.
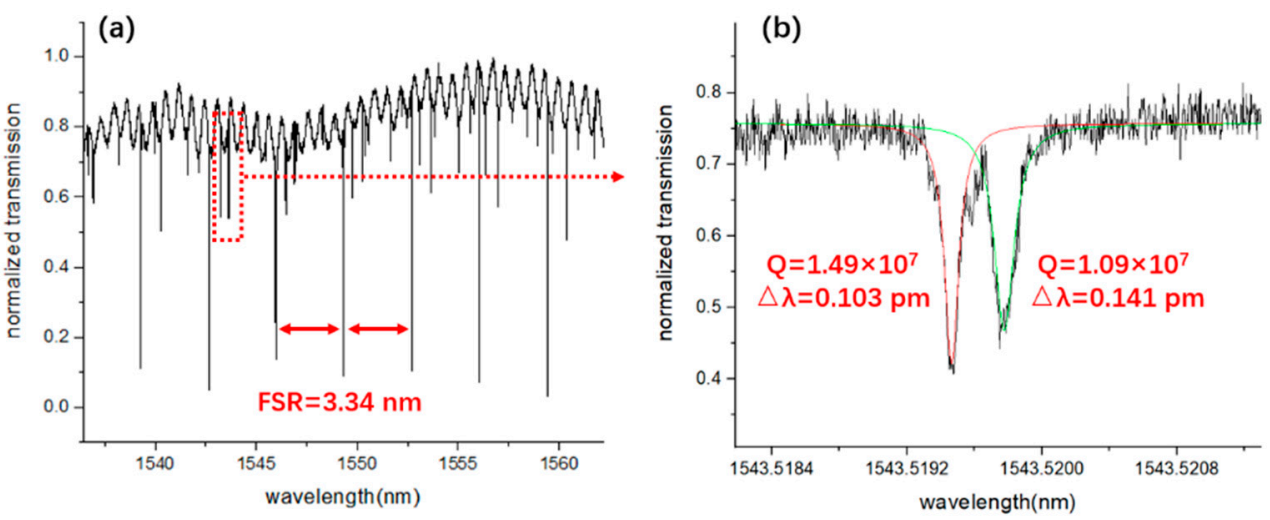

Figure 5. (a) Transmission spectrum of the LN microring resonator. (b) The Lorentz fitting of the splitting modes at the location of the red dotted box in Figure 4a reveals a Q-factor of $1.49 \times 10^{7}$ (red solid line) and $1.09 \times 10^{7}$ (green solid line), respectively.

Finally, we demonstrated that, in our scheme, the coupling efficiency as well as Qfactor can be tuned by changing the thickness of the $\mathrm{SiO}_{2}$ cladding layer, i.e., the vertical distance between the $\mathrm{Si}_{3} \mathrm{~N}_{4}$ waveguide and the $\mathrm{LN}$ microring. The distance was adjusted by precisely controlling the FIB etching depth of the $\mathrm{PECVD} \mathrm{SiO} 2$ layer. When the thickness of the $\mathrm{SiO}_{2}$ cladding layer was set to $1100 \mathrm{~nm}$, the coupling efficiency was relatively low, leading to the undercoupling between the $\mathrm{Si}_{3} \mathrm{~N}_{4}$ waveguide and the $\mathrm{LN}$ microring and giving rise to a high Q-factor of $6.6 \times 10^{6}$, as illustrated in Figure 6a,d. Subsequently, we adjusted the distance to $600 \mathrm{~nm}$, as shown in Figure 6b. The transmission loss of the light in the microring was close to the coupling loss between the waveguide and microring, which indicated that the critical coupling condition was reached. The deepest dip can be observed in the transmission curve presented in Figure 6e, and the Q-factor was $4.5 \times 10^{6}$. In general, the $Q$ factor should be higher for the critical coupling condition than that obtained in the undercoupling condition. Here, the slightly lower Q-factor measured in the critical coupling condition can be attributed to various imperfections in the fabrication process, which could influence the intrinsic $Q$ of the microring itself beneath the coupling $\mathrm{Si}_{3} \mathrm{~N}_{4}$ waveguide. When the distance was further reduced to $100 \mathrm{~nm}$, a higher coupling efficiency was reached at the strong over-coupling, whilst the $Q$ factor decreased to $2.6 \times 10^{6}$, as illustrated in Figure $6 \mathrm{c}, \mathrm{f}$. The $\mathrm{Q}$ factor that we mentioned here is loaded $\mathrm{Q}$ factor. 

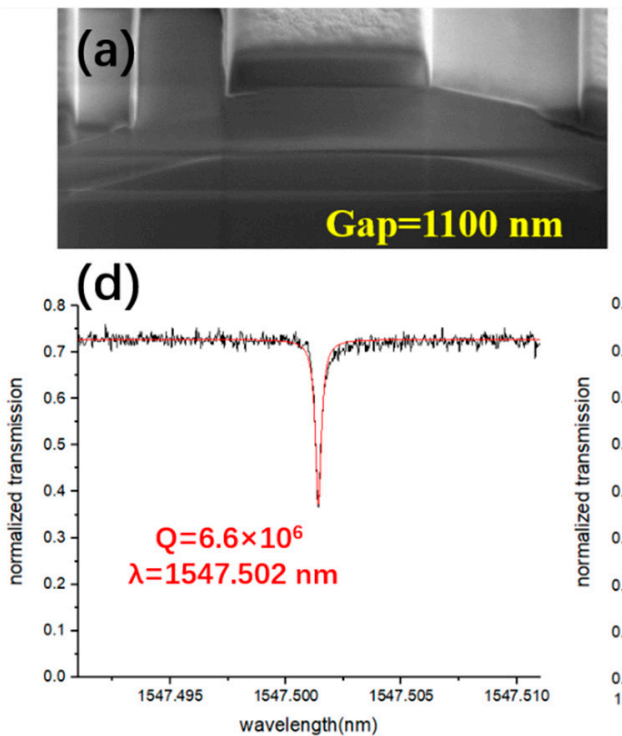

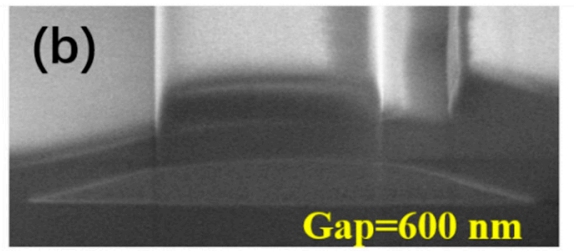

(e)

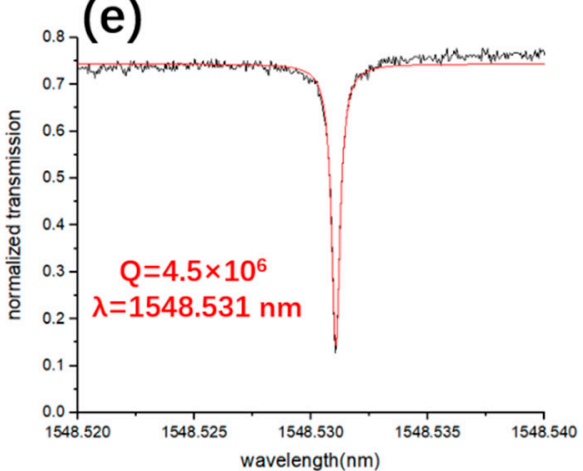

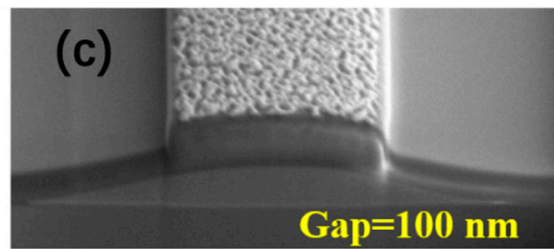

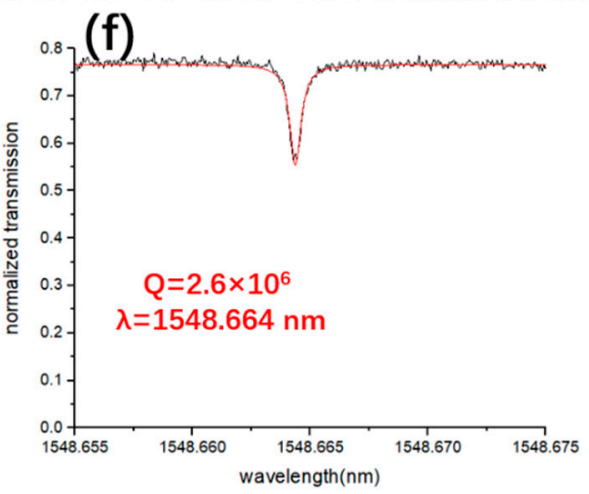

Figure 6. Sectional view SEM images of the coupling structures when the thickness of the $\mathrm{SiO}_{2}$ cladding layer are $1100 \mathrm{~nm}$ (a), $600 \mathrm{~nm}(\mathbf{b})$, and $100 \mathrm{~nm}$ (c), respectively. The Lorentz fitting of the modes of the structures in (a-c) are illustrated in $(\mathbf{d}-\mathbf{f})$, respectively.

\section{Conclusions}

To conclude, we have demonstrated efficient evanescent coupling between the crystalline $\mathrm{LN}$ microring resonator fabricated by PLACE and the $\mathrm{Si}_{3} \mathrm{~N}_{4}$ waveguide fabricated by FIB with a vertical configuration. By controlling the distance between the waveguide and microresonator, nearly critical coupling condition has been achieved with ultra-high $Q$ factors, i.e., the Q-factor of the fabricated LN microresonator was measured to be $1.49 \times 10^{7}$. Furthermore, we demonstrated that the coupling efficiency can be continuously tuned upon demand by varying the thickness of the $\mathrm{SiO}_{2}$ cladding layer. We should point out that the coupling efficiency can also be tuned by changing the relative position between the waveguide and the microring resonator in the horizontal plane. The scheme proposed in this work is also beneficial for large-scale PIC integration, as multiple microresonators can be remotely connected on a single chip using the same waveguide and the coupling efficiency can be individually tuned, as reasoned above. Thus, the scheme provides a promising photonic integration solution widely adopted by a broad range of LNOI photonic applications, which range from micro/nano-nonlinear optics and optical interconnect to on-chip artificial intelligence demonstration, etc.

Author Contributions: Conceptualization, Y.C.; methodology, Y.C. and W.C.; validation, J.Z. (Jianhao Zhang), M.W. (Ming Wang) and R.W.; formal analysis, J.Z. (Jianhao Zhang), R.W., W.C. and M.W. (Ming Wang); investigation, J.Z. (Jianhao Zhang), R.W., Z.F. and W.C.; resources, J.Z.(Jianhao Zhang), M.W. (Ming Wang), Z.F., R.W. and J.Z. (Junxia Zhou); data analyze, J.Z. (Jianhao Zhang), Y.L., W.C. and M.W. (Miao Wu); draft preparation, Y.C., W.C and J.Z. (Jianhao Zhang); supervision, W.C. and Y.C.; funding acquisition, Y.C. and W.C. All authors have read and agreed to the published version of the manuscript.

Funding: We acknowledge supports from National Key R\&D Program of China (2019YFA0705000), National Natural Science Foundation of China (11674340, 11734009, 11874154, 11874375, 61761136006, 61590934), the Strategic Priority Research Program of CAS (XDB16030300), Shanghai Municipal Science and Technology Major Project (2019SHZDZX01), and Key Research Program of Frontier Sciences, CAS (QYZDJ-SSW-SLH010).

Conflicts of Interest: The authors declare no conflict of interest. 


\section{References}

1. Boes, A.; Corcoran, B.; Chang, L.; Bowers, J.; Mitchell, A. Status and potential of lithium niobate on insulator (LNOI) for photonic integrated circuits. Laser Photon. Rev. 2018, 12, 1700256. [CrossRef]

2. Diziain, S.; Geiss, R.; Zilk, M.; Schrempel, F.; Kley, E.-B.; Tünnermann, A.; Pertsch, T. Mode analysis of photonic crystal L3 cavities in self-suspended lithium niobate membranes. Appl. Phys. Lett. 2013, 103, 251101. [CrossRef]

3. Zhang, M.; Wang, C.; Cheng, R.; Shams-Ansari, A.; Lončar, M. Monolithic ultra-high-Q lithium niobate microring resonator. Optica 2017, 4, 1536-1537. [CrossRef]

4. Hu, H.; Yang, J.; Gui, L.; Sohler, W. Lithium niobate-on-insulator (LNOI): Status and perspectives. Proc. SPIE 2012, 8431, 84311D.

5. Geiss, R.; Saravi, S.; Sergeyev, A.; Diziain, S.; Setzpfandt, F.; Schrempel, F.; Grange, R.; Kley, E.-B.; Tünnermann, A.; Pertsch, T. Fabrication of nanoscale lithium niobate waveguides for second-harmonic generation. Opt. Lett. 2015, 40, 2715-2718. [CrossRef]

6. Luo, R.; He, Y.; Liang, H.; Li, M.; Lin, Q. Semi-nonlinear nanophotonic waveguides for highly efficient second-harmonic generation. Laser Photon. Rev. 2019, 13, 1800288. [CrossRef]

7. Krasnokutska, I.; Tambasco, J.L.J.; Li, X.J.; Peruzzo, A. Ultra-low loss photonic circuits in lithium niobate on insulator. Opt. Express 2018, 26, 887-894. [CrossRef]

8. Lu, J.; Surya, J.B.; Liu, X.; Xu, Y.; Tang, H.X. Octave-spanning supercontinuum generation in nanoscale lithium niobate waveguides. Opt. Lett. 2019, 44, 1492-1495. [CrossRef]

9. He, M.; Xu, M.; Ren, Y.; Jian, J.; Ruan, Z.; Xu, Y.; Gao, S.; Sun, S.; Wen, X.; Zhou, L.; et al. High-performance hybrid silicon and lithium niobate Mach-Zehnder modulators for $100 \mathrm{Gbit} s-1$ and beyond. Nat. Photon. 2019, 13, 359-364. [CrossRef]

10. Chen, J.-Y.; Ma, Z.-H.; Sua, Y.M.; Li, Z.; Tang, C.; Huang, Y.-P. Ultra-efficient frequency conversion in quasi-phase-matched lithium niobate microrings. Optica 2019, 6, 1244-1245. [CrossRef]

11. Luo, R.; He, Y.; Liang, H.; Li, M.; Lin, Q. Highly tunable efficient second-harmonic generation in a lithium niobate nanophotonic waveguide. Optica 2018, 5, 1006-1011. [CrossRef]

12. Wu, R.; Zhang, J.; Yao, N.; Fang, W.; Qiao, L.; Chai, Z.; Lin, J.; Cheng, Y. Lithium niobate micro-disk resonators of quality factors above 107. Opt. Lett. 2018, 43, 4116-4119. [CrossRef] [PubMed]

13. Zhang, J.; Fang, Z.; Lin, J.; Zhou, J.; Wang, M.; Wu, R.; Gao, R.; Cheng, Y. Fabrication of crystalline microresonators of high quality factors with a controllable wedge angle on lithium niobate on insulator. Nanomaterials 2019, 9, 1218. [CrossRef] [PubMed]

14. Wu, R.B.; Wang, M.; Xu, J.; Qi, J.; Chu, W.; Fang, Z.W.; Zhang, J.H.; Zhou, J.X.; Qiao, L.L.; Chai, Z.F.; et al. Long low-loss-litium niobate on insulator waveguides with sub-nanometer surface roughness. Nanomaterials 2018, 8, 910. [CrossRef] [PubMed]

15. Jiang, W.C.; Lin, Q. Chip-scale cavity optomechanics in lithium niobate. Sci. Rep. 2016, 36920. [CrossRef] [PubMed]

16. Zhang, M.; Buscaino, B.; Wang, C.; Shams-Ansari, A.; Reimer, C.; Zhu, R.; Kahn, J.M.; Lončar, M. Broadband electro-optic frequency comb generation in a lithium niobate microring resonator. Nature 2019, 568, 373-377. [CrossRef] [PubMed]

17. Fang, Z.; Haque, S.; Farajollahi, S.; Luo, H.; Lin, J.; Wu, R.; Zhang, J.; Wang, Z.; Wang, M.; Cheng, Y.; et al. Polygon coherent modes in a weakly perturbed whispering gallery microresonator for efficient second harmonic, optomechanical, and frequency comb generations. Phys. Rev. Lett. 2020, 125, 173901. [CrossRef] [PubMed]

18. Wang, L.; Wang, C.; Wang, J.; Bo, F.; Zhang, M.; Gong, Q.; Lončar, M.; Xiao, Y.-F. High-Q chaotic lithium niobate microdisk cavity. Opt. Lett. 2018, 43, 2917. [CrossRef] [PubMed]

19. Wolf, R.; Breunig, I.; Zappe, H.; Buse, K. Scattering-loss reduction of ridge waveguides by sidewall polishing Opt. Express 2018, 26, 19815-19820. [CrossRef]

20. Pant, M.; Towsley, D.; Englund, D.; Guha, S. Percolation thresholds for photonic quantum computing. Nat. Commun. 2019, 10, 1070. [CrossRef] [PubMed] 